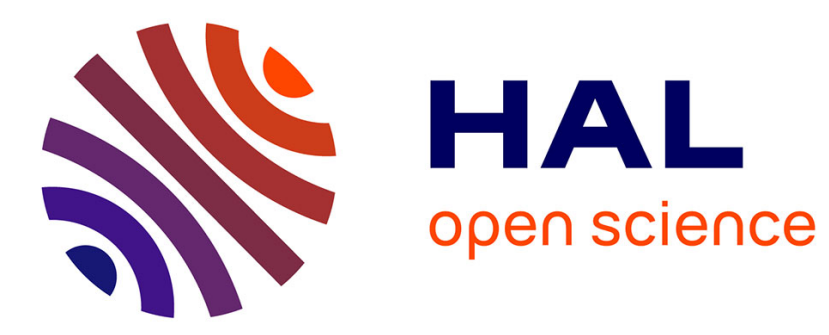

\title{
A woven reinforcement forming simulation method. Influence of the shear stiffness
}

Bassem Zouari, Jean-Luc Daniel, Philippe Boisse

\section{To cite this version:}

Bassem Zouari, Jean-Luc Daniel, Philippe Boisse. A woven reinforcement forming simulation method. Influence of the shear stiffness. Computers \& Structures, 2006, 84 (5-6), pp.351-363. 10.1016/j.compstruc.2005.09.031 . hal-00081069

\section{HAL Id: hal-00081069 \\ https://hal.science/hal-00081069}

Submitted on 17 Mar 2018

HAL is a multi-disciplinary open access archive for the deposit and dissemination of scientific research documents, whether they are published or not. The documents may come from teaching and research institutions in France or abroad, or from public or private research centers.
L'archive ouverte pluridisciplinaire HAL, est destinée au dépôt et à la diffusion de documents scientifiques de niveau recherche, publiés ou non, émanant des établissements d'enseignement et de recherche français ou étrangers, des laboratoires publics ou privés.

\section{다)(1) $(5$}

Distributed under a Creative Commons Attribution - NonCommercial| 4.0 International 


\title{
A woven reinforcement forming simulation method. Influence of the shear stiffness
}

\author{
Bassem Zouari ${ }^{\text {a }}$, Jean-Luc Daniel ${ }^{\text {a }}$, Philippe Boisse ${ }^{\text {b,* }}$ \\ ${ }^{a}$ Laboratoire de Mécanique de Systèmes et des Procédés, UMR CNRS 8106, ENSAM-ESEM, \\ 8 rue Léonard de Vinci, 45072 Orléans Cedex, France \\ ${ }^{\mathrm{b}}$ Laboratoire de Mécanique des Contacts et des Solides, UMR CNRS 5514, INSA de Lyon, \\ Bâtiment Jacquard, Rue Jean Capelle, 69621 Villeurbanne Cedex, France
}

\begin{abstract}
A simulation method is proposed for forming processes of fabrics used as reinforcements of composite materials. It uses specific finite elements made of woven material. The nodal interior loads are deduced from yarn tensile strain energy and woven cell shear energy. A picture frame shear test is presented. Optical measures permit to analyse the strains in yarns at microlevel for the different stages of the shear test. The influence of shearing in the formulation is studied on two forming simulations. It is shown that taking shear into account permits the appearance and the development of wrinkles when the locking angle is overcome.
\end{abstract}

Keywords: Fabric; Forming; Specific finite element; In-plane shear; Wrinkles

\section{Introduction}

Forming of composite materials exploits the relative movements of fibres made possible by the lack of cohesion of the matrix during the process. The matrix can be absent (forming of dry fabrics in the first stage of the R.T.M. process), be not polymerised yet (prepreg draped before polymerization of their thermoset matrix) or made fluid by heating (thermoplastic matrix). In this work, we consider the forming of dry woven reinforcements, but the study can be applied to the previous other cases when the reinforcements are continuous. A simulation tool permits the evaluation of the feasibility of a fabric forming process. It also gives the direction of the reinforcements after forming. This one conditions the mechanical behaviour of the final composite structure. In addition the angles between the warp and weft yarns also influence the permeability

\footnotetext{
Corresponding author.

E-mail address: Philippe.Boisse@insa-lyon.fr (P. Boisse).
}

of the reinforcement and thus the stage of filling by the resin. This stage is delicate and is the subject of many studies and development of specific codes [1]. For the simulation of dry fabrics forming, many codes have been developed on a geometrical approach (fishnet algorithm) [2-4]. These fast and effective methods make possible the calculation of the angles between the yarns in warp and weft directions in order to compare them with limiting values for which forming is not possible any more without wrinkles. On the other hand these methods do not take into account the mechanical behaviour of the reinforcements and the boundary conditions (blank holder load for example). Both of these two points affect (sometimes much) the obtained shape. An alternative to these geometrical methods is the use of finite element approaches. The domain can be considered as a continuous media, the mechanical behaviour of which models those of the fabric [5-7]. This is not easy especially because many stiffnesses of the fabric are very weak compared to the tensile rigidity. In the other hand the direction of the fibres (or yarns) must be followed very carefully $[6,7]$. 


\section{Nomenclature}

$a \quad$ side length of the picture frame

$A_{1}, A_{2}$ sections of warp and weft fibres

$C(\gamma)$ torque at warp and weft intersection

f exterior prescribed volume load

F load applied by the traction machine on the picture frame

$\mathbf{h}_{1}, \mathbf{h}_{2} \quad$ unit vectors in the warp and weft yarn directions

$\mathbf{h}^{1}, \mathbf{h}^{2}$ contravariant vectors related to $\mathbf{h}_{1}, \mathbf{h}_{2}$

$\operatorname{Int}(A)$ integer part of $A$

$\alpha, \beta \quad$ indexes equal to 1 or 2

$k, k_{1}, k_{2}$ shear rigidities

${ }^{p} l_{\alpha} \quad$ length of the fibre $p$ in the direction of $\mathbf{h}_{\alpha}$

$n_{t}, n_{c} \quad$ number of weft and warp fibres in the element

$N_{t}, N_{c}$ number of weft and warp fibres in the fabric specimen

$N^{k}, k=1,4$ interpolation functions of a quadrangle

$s \quad$ range of the degree of freedom in the element $s \in\{1, \ldots, 12\}$

t exterior prescribed surface load applied on $\Gamma_{t}$

$\mathbf{T}=T^{\alpha \beta} \mathbf{h}_{\alpha} \otimes \mathbf{h}_{\beta}$ tension tensor

$T_{\text {ext }}(\boldsymbol{\eta})$ virtual work of exterior loads

$T_{\text {ext }}^{\mathrm{e}}(\boldsymbol{\eta})$ elementary virtual work of exterior loads

$T_{\text {int }}(\boldsymbol{\eta})$ virtual work of interior loads

$T_{\text {int }}^{\mathrm{e}}(\boldsymbol{\eta})$ elementary virtual work of interior loads

u displacement

ù velocity

ü acceleration

In this paper an alternative is proposed where finite elements, specific to woven materials, are constructed. They are composed of woven yarns in tension and shear and the nodal interior loads are obtained from yarn and woven load strain energy. This permits an approach that is closed from the physics. The tensile and the shear mechanical behaviour is naturally introduce from experimental tests. One goal of this paper is to show the influence of the shear stiffness in the formulation. The computations of forming processes are made taking or not the shear strain energy into account. It is shown that the main improvement due to shear is the description of wrinkles when the shear limit angle is overcome.

\section{Experimental analysis}

The mechanical behaviour of a woven composite reinforcement is special because of the possible motions between fibres and yarns. Consequently most of the rigidities can be neglected as regard to tension stiffness. In the present work, tension stiffness will be considered and in plane shear stiffness will be (possibly) added. It will be shown that this shear stiffness is important in some cases. Bending rigidity will be neglected.
$\mathbf{V}_{\mathrm{C}} \quad$ velocity of the traction machine crossbar $\boldsymbol{\varepsilon}=\varepsilon_{\alpha \beta} \mathbf{h}^{\alpha} \otimes \mathbf{h}^{\beta}$ symmetrical gradient strain tensor

$\gamma \quad$ relative rotation between warp and weft fibres (or shear angle)

$\dot{\gamma} \quad$ relative rotation speed between warp and weft fibres

$\gamma_{c} \quad$ shear limit angle

$p_{\gamma}(\boldsymbol{\eta}) \quad$ rotation between warp and weft yarns related to the virtual displacement field $\boldsymbol{\eta}$ for the woven cell $p$

$\boldsymbol{\eta} \quad$ virtual displacement field with $\boldsymbol{\eta}=0$ on the part of the frontier of $\Omega$ with prescribed displacements $\Gamma_{u}$

$\eta_{\mathrm{s}} \quad$ component number $n$ of the virtual displacement

$\theta_{0}=\left(\mathbf{g}_{10}, \mathbf{g}_{20}\right)$ angle between warp and weft yarns at the start of the time step

$\theta=\left(\mathbf{g}_{1}, \mathbf{g}_{2}\right)$ angle between warp and weft yarns in the virtual configuration

$\rho \quad$ mass per volume of the fabric

$\boldsymbol{\sigma}=\sigma^{\alpha \beta} \mathbf{h}_{\alpha} \otimes \mathbf{h}_{\beta}$ Cauchy stress tensor

$\Gamma_{t} \quad$ part of the frontier of $\Omega$ with prescribed loads

$\Gamma_{u} \quad$ part of the frontier of $\Omega$ with prescribed displacements

$\xi_{1}, \xi_{2}$ coordinates in the reference element

$\Omega \quad$ current fabric domain under consideration

The behaviour of fabric reinforcements in biaxial tension has been studied in $[8,9]$ and recently in $[10,11]$. In this section, an experimental study of the reinforcement in plane shear is considered. There are mainly three experimental methods for the study of fabric shear deformation. In the first method, parallel and opposite displacements are applied to two opposite sides of a rectangular fabric specimen. The strain state corresponds to a shearing case superposed with tensions (Fig. 1(a)). Many parameters influence the measures, as the report of elongation of the fabric specimen and the applied transverse force. The measurement dispersions in this case can be important. Kawabata et al.

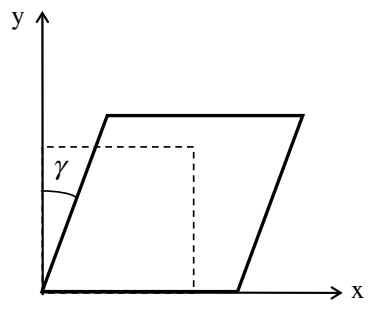

a

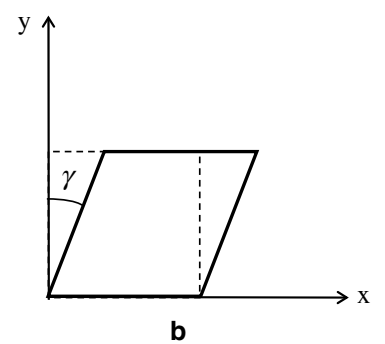

b
Fig. 1. Shear kinematics: (a) shearing + tensions; (b) simple shearing. 
[12] developed a device imposing a simple shear state to a woven fabric specimen (KES-F) (Fig. 1(b)). The second type of shear tests on woven reinforcement is the FAST test (Fabric Assurance by Simple Testing) or "bias test". Its aim is to prescribe a tensile load to the specimen in a direction different from the yarns ones, typically to $45^{\circ}$. Several works [13-16] have shown that the field of deformation is not uniform within the specimen. In fact, only the central zone is subject to a pure shear deformation. This test is used frequently because of its simplicity. During this test a slip between the yarns can happen, which is contrary to the hypotheses of a continuum for the fabric. The third method consists in the use of a hinged frame called picture frame. We used this technique that is described in the following paragraph to characterise the shear behaviour of woven fabrics.

\subsection{Experimental analysis by picture frame shear test}

The picture frame shear test consists in clamping a fabric on a hinged frame whose directions are those of the fabric yarns (Fig. 2) [17-19]. Different studies have showed that this device is the most satisfactory to impose a homogeneous shear deformation in a woven reinforcement $[20,21]$. The frame is installed on a conventional tensile machine. A pre-tension is applied on the fabric yarns before shearing. The active surface of the presented test specimen is $200 \times 200 \mathrm{~mm}^{2}$. The specimen under consideration is a glass plain weave (T6) presenting the following features: yarn density $=0.25$, crimp: $S=0.5 \%$, surface density: $W=600 \mathrm{~g} / \mathrm{m}^{2}$, linear density: $w=$ 1200 tex $(1$ tex $=1 \mathrm{~g} / \mathrm{km})$. The curve giving the evolution of the applied load versus the displacement (Fig. 3) can be divided into three zones. In the first part the slope is small and the shear rigidity is very weak. In the second zone, the shear rigidity increases and becomes very important in the third part. Optical measurements are used at macro- and micro-level in order to understand the shear deformation mechanisms operating during these three phases. They are described in the following paragraph.

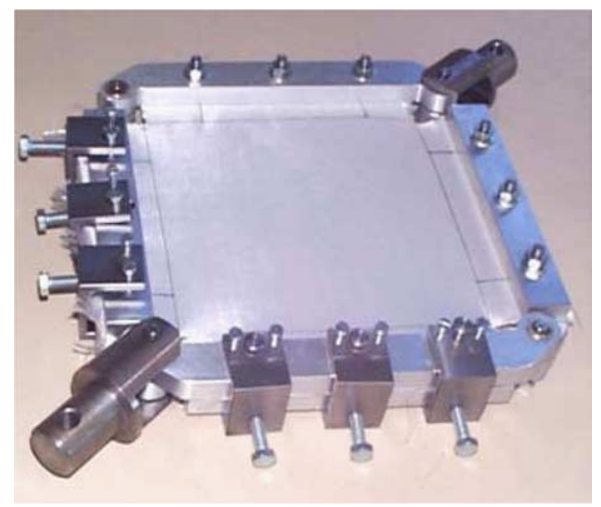

Fig. 2. Shear picture frame.

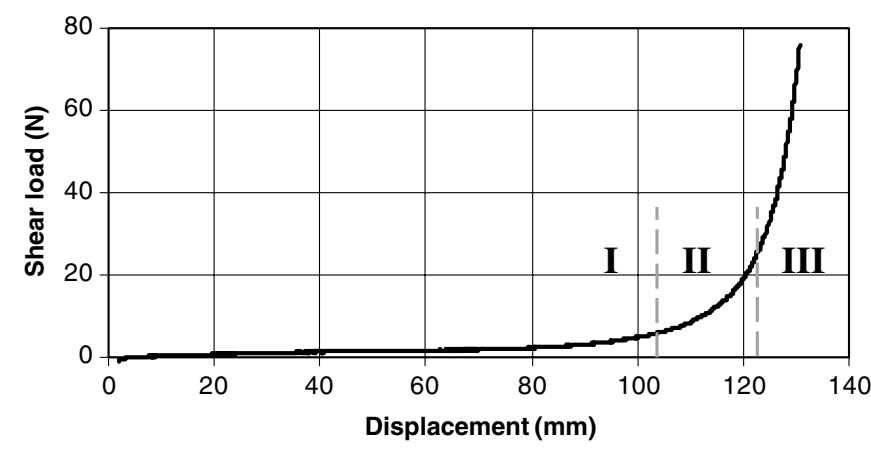

Fig. 3. Shear load versus the displacement.

\subsection{Optical measurements of deformation}

An optical system (Fig. 4) acquires pictures of the whole frame (macroscale) or only of a few number of yarns $\left(12.4 \times 9.5 \mathrm{~mm}^{2}\right)$. The measure domain can be restricted to a unique yarn (microscale). Two pictures (macro and micro) are taken simultaneously using cameras at both sides of the specimen. These pictures are used to calculate the displacement and strain fields by applying a digital image correlation method $[22,23]$. It permits to verify the homogeneity of the shear deformation in the specimen [21]. Fig. 5(a) shows the displacements at the microscale (inside the yarn) during the phase 1 . The average displacement is subtracted to the measured ones. In this phase, the yarns are submitted to a rigid body rotation. There is no shearing inside the yarns. The global fabric shear is due to relative parallel displacement of yarns. This explains that the fabric shearing corresponds to weak loads since only the friction due to rotation between warp and weft yarns is opposed to the distortion. The transition to the zone 2 corresponds to the limit angle or shear locking angle. After this angle, the yarns become in contact with their neighbours and are laterally compressed (Fig. 5(b)), first partially (zone 2) then completely (zone 3) (Fig. 5(c)). This explains the very fast

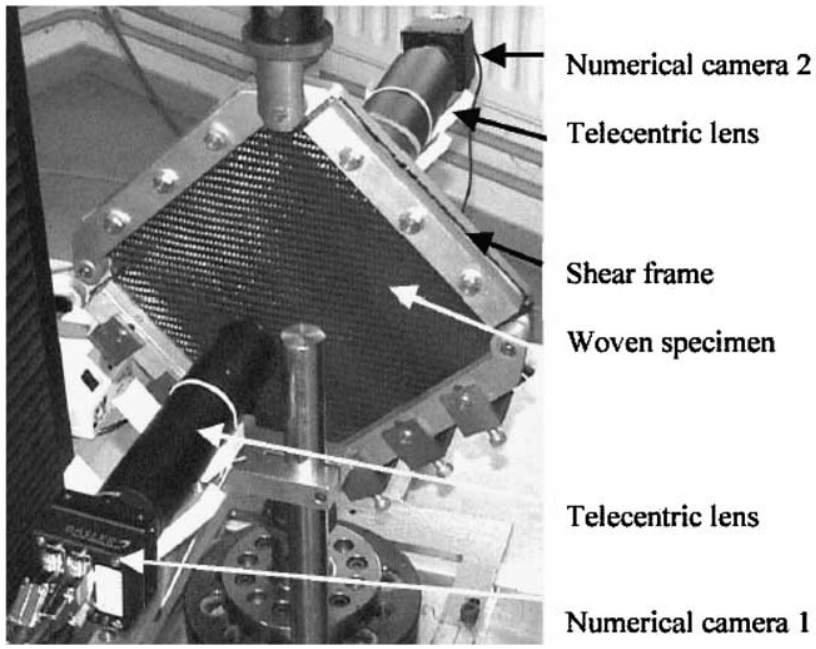

Fig. 4. Shear frame device equipped with the optical system. 


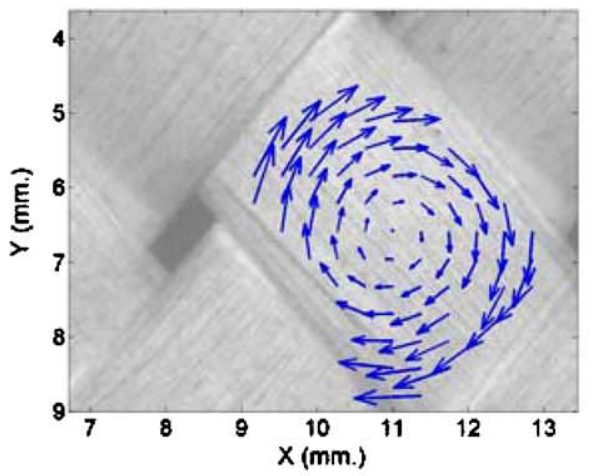

a

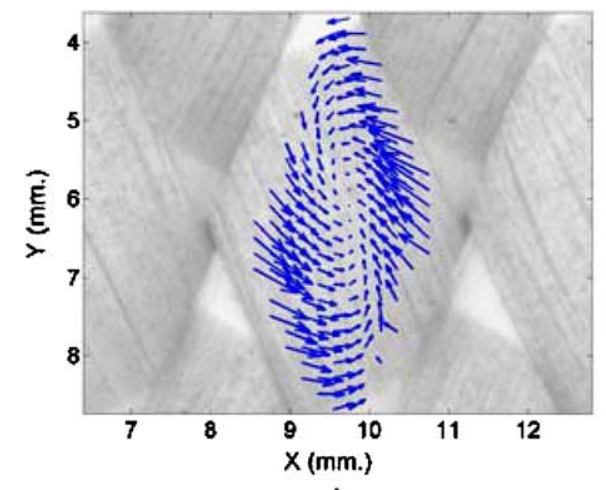

b

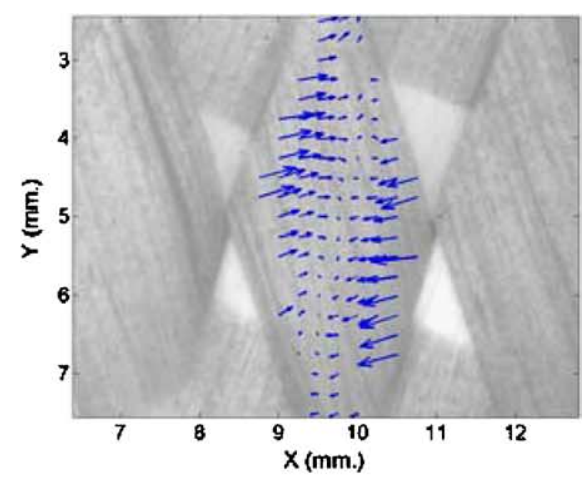

c

Fig. 5. Displacement field in a yarn during the three phases of shear.

increase of the shearing force and therefore of the shear rigidity. In practice in the zone 3 , wrinkles appear caused by the shear locking.

\section{Finite element for the simulation of woven reinforcements sheet forming}

Finite elements developed to simulate the sheet forming of woven reinforcements are presented in this section. The element formulation taking only the tension rigidity in warp and weft directions into account is reminded first $[24,25]$. Then, the plane shear behaviour and its implementation in the finite elements are presented.

\subsection{Tension behaviour}

\subsubsection{Elementary cell}

It has been shown by some experiments [24] that it can be assumed that there is no translation sliding between warp and weft yarns in a usual forming process. This justifies the use of classical lagrangian finite elements for woven reinforcement sheet forming modelling. The fabric finite element that has been developed by the authors $[24,25,27]$ and which take into account only the two rigidities of traction in warp and weft directions is briefly presented in the following paragraph.

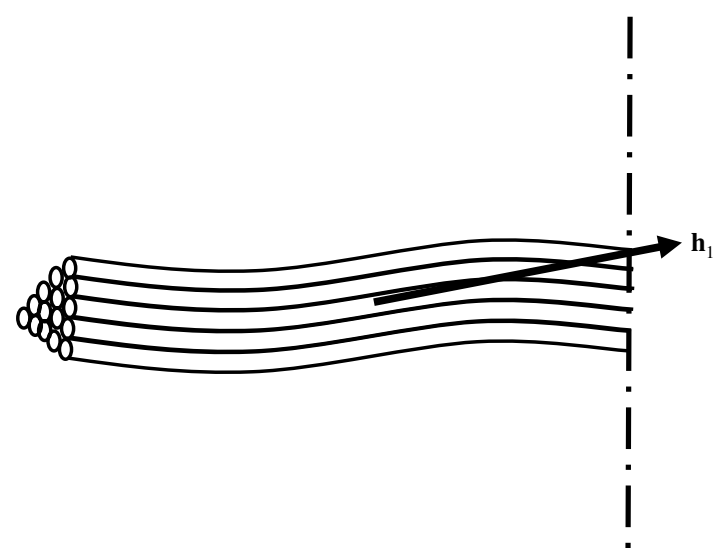

In the present study the tensile behaviours of warp and weft yarns are assumed to be uncoupled. An approach taking interaction between the two direction can be found in $[28,29]$. If $\mathbf{h}_{1}$ is the unit vector in the fibre direction (see Fig. 6), the Cauchy stress tensor in a yarn in tension is

$\boldsymbol{\sigma}=\sigma^{11} \mathbf{h}_{1} \otimes \mathbf{h}_{1}$

The tension tensor, that has more physical significance in the case of fabrics than the stress tensor, is defined as

$\mathbf{T}=T^{11} \mathbf{h}_{1} \otimes \mathbf{h}_{1}$

$T^{11}=\int_{\mathrm{A}_{1}} \sigma^{11} \mathrm{~d} S$

\subsubsection{Finite element formulation}

Let us consider a domain $\Omega$ composed by $n$ yarns, the principle of the virtual works in the dynamic case can be written as

$$
\forall \boldsymbol{\eta} / \boldsymbol{\eta}=0 \text { on } \Gamma_{u}
$$

$\sum_{p=1}^{\text {fibres }} \int_{{ } l_{1}}{ }^{p} \varepsilon_{11}(\boldsymbol{\eta})^{p} T^{11} \mathrm{~d} l-T_{\text {ext }}(\boldsymbol{\eta})=\int_{\Omega} \rho \ddot{\mathbf{u}} \cdot \boldsymbol{\eta} \mathrm{d} V$

The virtual work of the exterior loads $T_{\text {ext }}(\boldsymbol{\eta})$ is

$T_{\text {ext }}(\boldsymbol{\eta})=\int_{\Omega} \mathbf{f} \cdot \boldsymbol{\eta} \mathrm{d} V+\int_{\Gamma_{t}} \mathbf{t} \cdot \boldsymbol{\eta} \mathrm{d} S$

The presented finite element is an isoparametric bilinear 4 nodes element. The natural directions of the element coincide with the warp and weft directions (Fig. 7) for numerical efficiency [30]. The element contains $n_{c}$ warp and $n_{t}$ weft yarns. The material co-ordinate system $\left(\xi_{1}, \xi_{2}\right)$, in the warp and weft directions, defines a covariant material base $\left(\mathbf{g}_{1}, \mathbf{g}_{2}\right)$ as well as the contravariant associated base

$\mathbf{g}_{\alpha}=\frac{\partial \mathbf{x}}{\partial \xi_{\alpha}}, \quad \mathbf{g}_{\alpha} \cdot \mathbf{g}^{\beta}=\delta_{\alpha}^{\beta}$

The virtual work of interior loads is

$T_{\text {int }}^{\mathrm{e}}(\boldsymbol{\eta})=\sum_{p=1}^{n_{t}} \int_{{ }^{2} l_{1}}{ }^{p} \varepsilon_{11}(\boldsymbol{\eta})^{\mathrm{P}} T^{11} \mathrm{~d} l+\sum_{p=1}^{n_{c}} \int_{{ }^{2} l_{2}}{ }^{p} \varepsilon_{22}(\boldsymbol{\eta})^{p} T^{22} \mathrm{~d} l$

Fig. 6. Direction of the yarn. 


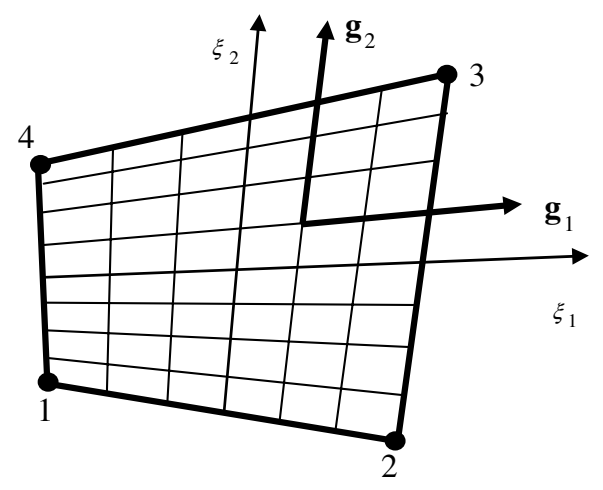

Fig. 7. Woven finite element.

The components of the symmetrical gradient tensor are considered in $\mathbf{h}^{1}, \mathbf{h}^{2}$ and $\mathbf{g}^{1}, \mathbf{g}^{2}$

$\nabla^{s} \boldsymbol{\eta}=\varepsilon_{\alpha \beta} \mathbf{h}^{\alpha} \otimes \mathbf{h}^{\beta}=\bar{\varepsilon}_{\alpha \beta} \mathbf{g}^{\alpha} \otimes \mathbf{g}^{\beta}$

The components $\bar{\varepsilon}_{\alpha \alpha}$ are interpolated in function of the virtual displacements

$\bar{\varepsilon}_{\alpha \alpha}=\frac{\partial \boldsymbol{\eta}}{\partial \xi_{\alpha}} \cdot \mathbf{g}_{\alpha}=\frac{\partial N^{k}}{\partial \xi_{\alpha}}\left(\mathbf{g}_{\alpha}\right)_{m} \eta_{s}=B_{\alpha \alpha s} \eta_{s}$

where $k=\operatorname{Int}\left(\frac{s+2}{3}\right), m=s-3(k-1), s$ is the range of the degree of freedom in the element and $\operatorname{Int}(A)$ is the integer part of $A$.

The elementary virtual interior work can be written

$$
\begin{aligned}
T_{\text {int }}^{\mathrm{e}}(\boldsymbol{\eta})= & \eta_{s} \sum_{p=1}^{n_{t}} \int_{p l_{1}} \frac{1}{\left\|\mathbf{g}_{1}\right\|^{2}}{ }^{p} B_{11 s}^{p} T^{11} \mathrm{~d} s \\
& \left.+\sum_{p=1}^{n_{c}} \int_{p l_{1}} \frac{1}{\left\|\mathbf{g}_{2}\right\|^{2}} B_{22 s}^{p} T^{22} \mathrm{~d} s\right)=\eta_{s}\left(F_{\text {int }}^{\mathrm{e}}\right)_{s}
\end{aligned}
$$

The quantities present in the interior loads do not depend of $\xi_{\alpha}$. Taking into account that $\mathrm{d} s=\left\|\mathbf{g}_{\alpha}\right\| \mathrm{d} \xi_{\alpha}$, the nodal components of the interior loads for warp and weft yarns are

$\left(F_{\text {int }}^{\mathrm{e}}\right)_{s}=\sum_{p=1}^{n_{t}}{\frac{1}{\left\|\mathbf{g}_{1}\right\|}}^{p} B_{11 s}^{p} T^{11}+\sum_{p=1}^{n_{c}}{\frac{1}{\left\|\mathbf{g}_{2}\right\|}}^{p} B_{22 s}^{p} T^{22}$

Because of the bilinear interpolation function of the element, it is not necessary in the previous equation to sum over all the yarns. There is two "Gauss fibres" where Eq. (11) is equivalent to

$\left(F_{\mathrm{int}}^{\mathrm{e}}\right)_{s}=\sum_{p=1}^{2} n_{t} B_{11 s} T^{11} \frac{1}{\left\|\mathbf{g}_{1}\right\|}+\sum_{p=1}^{2} n_{c} B_{22 s} T^{22} \frac{1}{\left\|\mathbf{g}_{2}\right\|}$

The positions of these two Gauss fibres are

$\xi_{2}= \pm \sqrt{\frac{1}{3} \frac{n_{t}^{2}-1}{n_{t}^{2}}}, \quad \xi_{1}= \pm \sqrt{\frac{1}{3} \frac{n_{c}^{2}-1}{n_{c}^{2}}}$

for the warp and weft directions, respectively [26].

The element is implemented in an explicit code, consequently the nodal interior loads $F_{\text {int }}^{\mathrm{e}}$ is the only elementary quantity needed for the computation. Details on this (standard) explicit approach can be found in Ref. [29].

\subsection{Taking in-plane shear into account}

The observations made in the shear tests on the woven reinforcements, show that the fabric deformation is the result of a relative rotation between the warp and weft yarns and not of a fibres shearing [31] (Fig. 5). The tensile mechanical behaviour of the fabric presented in Section 3.1 is completed by adding a shear rigidity to every yarn crossover [32]. The relative rotations of the warp and weft yarns lead to a torque applied at the yarn intersections. The value of this shear torque for a shear angle is experimentally given by the picture frame test. To find the value of this resistant torque, it is assumed that the fibres remain straight during the test [12]. Consequently, all the angles (between warp and weft) and all the torques at the intersections are equal over the frame. The dissipated power is assumed to be small. The power provided by the traction machine and the power absorbed by the warp and weft intersections are supposed to be equal. Using the notations of Fig. 8, we get

$P=\mathbf{F} \cdot \mathbf{V}_{\mathrm{C}}=N_{c} N_{t} C(\gamma) \dot{\gamma}$

The elementary torque according to the shear angle is given by

$C(\gamma)=\frac{a}{N_{c} N_{t}} \frac{\sqrt{2}}{2}\left(\cos \frac{\gamma}{2}-\sin \frac{\gamma}{2}\right) F\left(U_{\mathrm{C}}(\gamma)\right)$

Fig. 9 shows the torque according to the shear angle. This curve can be divided into three zones in such a way as the load-displacement curve (Fig. 3). The torque value is small at the beginning and becomes important after the locking angle. The shear and tension behaviour are assumed to be uncoupled. In a first approach, the torque-angle curve is modelled by two straight segments of different slope. Thus, the shear fabric behaviour is characterised by two rigidities $k_{1}, k_{2}$ and a limit angle $\gamma_{c}$. The following relation between the torque and the shear angle is assumed:

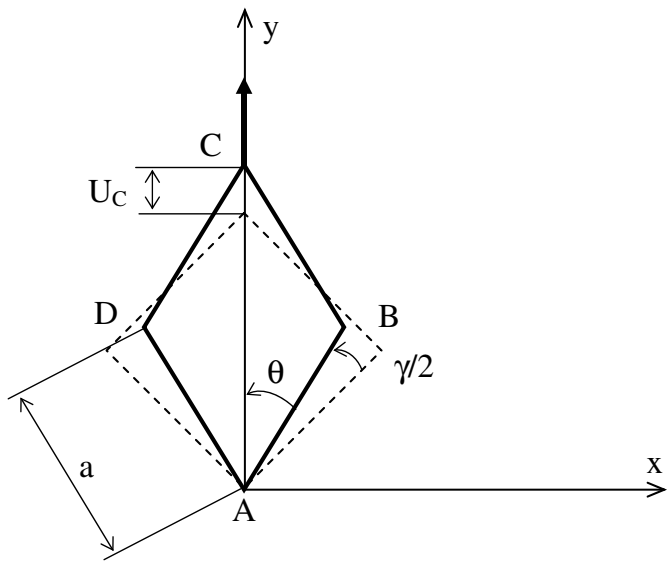

Fig. 8. Parameters and kinematics of the shear picture frame. 


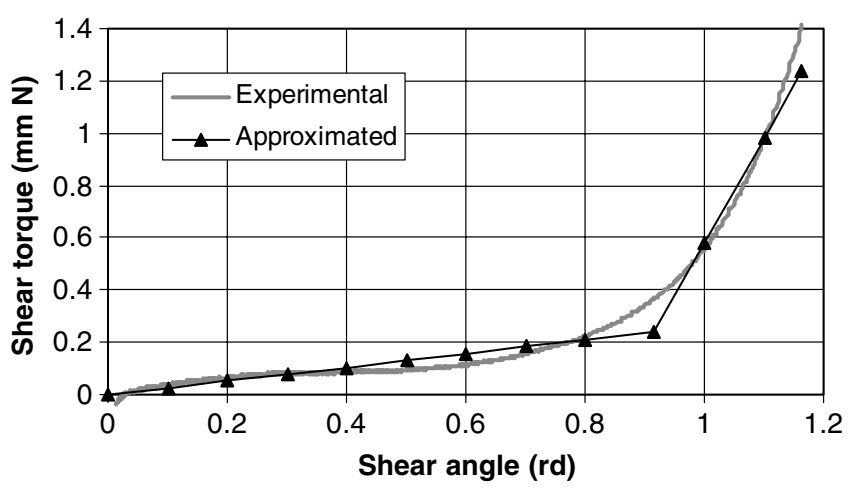

Fig. 9. Shear torque versus shear angle.

$$
\left\{\begin{array}{l}
C(\gamma)=k_{1} \gamma \quad \text { if } \gamma \leqslant \gamma_{c} \\
C(\gamma)=k_{2}\left(\gamma-\gamma_{c}\right)+k_{1} \gamma_{c} \quad \text { if } \gamma>\gamma_{c}
\end{array}\right.
$$

In the case of a glass plain weave (T6), the three following values of the shear behaviour are identified:

$k_{1}=0.26 \mathrm{mmN} / \mathrm{rd}, \quad k_{2}=4.03 \mathrm{mmN} / \mathrm{rd}$,

$\gamma_{c}=0.9148 \mathrm{rd}$

\subsection{Implementation of the shear stiffness in the fabric finite element}

The introduction of the shear contribution in the expression of the virtual works in dynamics leads to:

$$
\begin{aligned}
& \sum_{p=1}^{\text {fibres }} \int_{{ }^{p} l_{1}}{ }^{p} \varepsilon_{11}(\boldsymbol{\eta})^{p} T^{11} \mathrm{~d} l+\sum_{p=1}^{\text {crossovers }}{ }^{p} C^{p} \gamma(\boldsymbol{\eta})-T_{\text {ext }}(\boldsymbol{\eta}) \\
& \quad=\int_{\Omega} \rho \ddot{\mathbf{u}} \cdot \boldsymbol{\eta} \mathrm{d} V
\end{aligned}
$$

Within a finite element approach, the elementary virtual work of the interior loads is

$$
\begin{aligned}
T_{\text {int }}^{\mathrm{e}}(\boldsymbol{\eta})= & \left.\eta_{s} \sum_{p=1}^{n_{t}} \frac{1}{\left\|\mathbf{g}_{1}\right\|}{ }^{p} B_{11 s}{ }^{p} T^{11}+\sum_{p=1}^{n_{c}} \frac{1}{\left\|\mathbf{g}_{2}\right\|}{ }^{p} B_{22 s}{ }^{p} T^{22}\right) \\
& +\sum_{p=1}^{n_{t} n_{c}}{ }^{p} C^{p} \gamma(\boldsymbol{\eta})
\end{aligned}
$$

As it is defined in (17) and (18), the shear contribution does not permit to express the interior virtual load work as a scalar product of the virtual displacement vector and the interior load vector. In this goal, first order development of ${ }^{p} \gamma(\boldsymbol{\eta})$ is performed. The use of an explicit resolution scheme requires (for stability reasons) a very small time step. Consequently the rotations between two consecutive configurations are small, that justifies the linearisation.

The rotation between warp and weft yarns following the virtual displacement $\boldsymbol{\eta}$ is:

$\gamma(\boldsymbol{\eta})=\arccos \left(\frac{\mathbf{g}_{1} \cdot \mathbf{g}_{2}}{\left\|\mathbf{g}_{1}\right\|\left\|\mathbf{g}_{2}\right\|}\right)-\arccos \left(\frac{\mathbf{g}_{10} \cdot \mathbf{g}_{20}}{\left\|\mathbf{g}_{10}\right\|\left\|\mathbf{g}_{20}\right\|}\right)$ $\mathbf{g}_{\alpha 0}=\frac{\partial \mathbf{x}_{0}}{\partial \xi_{\alpha}}$ and $\mathbf{g}_{\alpha}=\frac{\partial \mathbf{x}}{\partial \xi_{\alpha}}=\frac{\partial(\mathbf{x}+\mathbf{\eta})}{\partial \xi_{\alpha}}$ are the covariant vectors in the present configuration and the virtual configuration.

$$
\begin{aligned}
\gamma(\boldsymbol{\eta})= & \arccos \left(\frac{\left(\mathbf{g}_{10}+\frac{\partial \boldsymbol{\eta}}{\partial \xi_{1}}\right) \cdot\left(\mathbf{g}_{20}+\frac{\partial \boldsymbol{\eta}}{\partial \xi_{2}}\right)}{\left\|\mathbf{g}_{10}+\frac{\partial \boldsymbol{\eta}}{\partial \xi_{1}}\right\|\left\|\mathbf{g}_{20}+\frac{\partial \boldsymbol{\eta}}{\partial \xi_{2}}\right\|}\right) \\
& -\arccos \left(\frac{\mathbf{g}_{10} \cdot \mathbf{g}_{20}}{\left\|\mathbf{g}_{10}\right\|\left\|\mathbf{g}_{20}\right\|}\right)
\end{aligned}
$$

We have

$\left\|\mathbf{g}_{\alpha 0}+\frac{\partial \boldsymbol{\eta}}{\partial \xi_{\alpha}}\right\|=\sqrt{\left\|\mathbf{g}_{\alpha 0}\right\|^{2}+\left\|\frac{\partial \boldsymbol{\eta}}{\partial \xi_{\alpha}}\right\|^{2}+2 \mathbf{g}_{\alpha 0} \cdot \frac{\partial \boldsymbol{\eta}}{\partial \xi_{\alpha}}}$

Neglecting the terms of second order in $\boldsymbol{\eta}$ and making a first order development, we get

$$
\begin{aligned}
\left\|\mathbf{g}_{\alpha 0}+\frac{\partial \boldsymbol{\eta}}{\partial \xi_{\alpha}}\right\| & \simeq\left\|\mathbf{g}_{\alpha 0}\right\| \sqrt{1+2 \frac{\mathbf{g}_{\alpha 0}}{\left\|\mathbf{g}_{\alpha 0}\right\|^{2}} \cdot \frac{\partial \boldsymbol{\eta}}{\partial \xi_{\alpha}}} \\
& \left.\simeq\left\|\mathbf{g}_{\alpha 0}\right\| 1+\frac{\mathbf{g}_{\alpha 0}}{\left\|\mathbf{g}_{\alpha 0}\right\|^{2}} \cdot \frac{\partial \boldsymbol{\eta}}{\partial \xi_{\alpha}}\right)
\end{aligned}
$$

and

$$
\begin{aligned}
& \left(\mathbf{g}_{10}+\frac{\partial \boldsymbol{\eta}}{\partial \xi_{1}}\right) \cdot\left(\mathbf{g}_{20}+\frac{\partial \boldsymbol{\eta}}{\partial \xi_{2}}\right) \\
& \simeq \mathbf{g}_{10} \cdot \mathbf{g}_{20}+\mathbf{g}_{20} \cdot \frac{\partial \boldsymbol{\eta}}{\partial \xi_{1}}+\mathbf{g}_{10} \cdot \frac{\partial \boldsymbol{\eta}}{\partial \xi_{2}}
\end{aligned}
$$

Consequently

$$
\begin{aligned}
& \frac{\left(\mathbf{g}_{10}+\frac{\partial \boldsymbol{\eta}}{\partial \xi_{1}}\right) \cdot\left(\mathbf{g}_{20}+\frac{\partial \boldsymbol{\eta}}{\partial \xi_{2}}\right)}{\left\|\mathbf{g}_{10}+\frac{\partial \boldsymbol{\eta}}{\partial \xi_{1}}\right\|\left\|\mathbf{g}_{20}+\frac{\partial \boldsymbol{\eta}}{\partial \xi_{2}}\right\|} \\
& \simeq \frac{\mathbf{g}_{10} \cdot \mathbf{g}_{20}+\mathbf{g}_{20} \cdot \frac{\partial \boldsymbol{\eta}}{\partial \xi_{1}}+\mathbf{g}_{10} \cdot \frac{\partial \boldsymbol{\eta}}{\partial \xi_{2}}}{\left\|\mathbf{g}_{10}\right\|\left\|\mathbf{g}_{20}\right\|\left(1+\frac{\mathbf{g}_{10}}{\left\|\mathbf{g}_{10}\right\|^{2}} \cdot \frac{\partial \boldsymbol{\eta}}{\partial \xi_{1}}\right)\left(1+\frac{\mathbf{g}_{20}}{\left\|\mathbf{g}_{20}\right\|^{2}} \cdot \frac{\partial \boldsymbol{\eta}}{\partial \xi_{2}}\right)}
\end{aligned}
$$

Neglecting the terms of the second order in $\boldsymbol{\eta}$, we get

$$
\begin{aligned}
& \frac{\left(\mathbf{g}_{10}+\frac{\partial \boldsymbol{\eta}}{\partial \xi_{1}}\right) \cdot\left(\mathbf{g}_{20}+\frac{\partial \boldsymbol{\eta}}{\partial \xi_{2}}\right)}{\left\|\mathbf{g}_{10}+\frac{\partial \boldsymbol{\eta}}{\partial \xi_{1}}\right\|\left\|\mathbf{g}_{20}+\frac{\partial \boldsymbol{\eta}}{\partial \xi_{2}}\right\|} \\
& \simeq \frac{\left(\mathbf{g}_{10} \cdot \mathbf{g}_{20}+\mathbf{g}_{20} \cdot \frac{\partial \boldsymbol{\eta}}{\partial \xi_{1}}+\mathbf{g}_{10} \cdot \frac{\partial \boldsymbol{\eta}}{\partial \check{\xi}_{2}}\right)-\left(\mathbf{g}_{10} \cdot \mathbf{g}_{20}\right)\left(\frac{\mathbf{g}_{10}}{\left\|\mathbf{g}_{10}\right\|^{2}} \cdot \frac{\partial \boldsymbol{\eta}}{\partial \xi_{1}}+\frac{\mathbf{g}_{20}}{\left\|\mathbf{g}_{20}\right\|^{2}} \cdot \frac{\partial \boldsymbol{\eta}}{\partial \xi_{2}}\right)}{\left\|\mathbf{g}_{10}\right\|\left\|\mathbf{g}_{20}\right\|}
\end{aligned}
$$

We note $\theta_{0}=\left(\mathbf{g}_{10}, \mathbf{g}_{20}\right)$ the angle between warp and weft yarns at the beginning of the time step

$$
\begin{aligned}
\frac{\left(\mathbf{g}_{10}+\frac{\partial \boldsymbol{\eta}}{\partial \xi_{1}}\right) \cdot\left(\mathbf{g}_{20}+\frac{\partial \boldsymbol{\eta}}{\partial \xi_{2}}\right)}{\left\|\mathbf{g}_{10}+\frac{\partial \boldsymbol{\eta}}{\partial \xi_{1}}\right\|\left\|\mathbf{g}_{20}+\frac{\partial \boldsymbol{\eta}}{\partial \xi_{2}}\right\|} & \simeq \cos \left(\theta_{0}\right)+\frac{\left(\mathbf{g}_{20} \cdot \frac{\partial \boldsymbol{\eta}}{\partial \xi_{1}}+\mathbf{g}_{10} \cdot \frac{\partial \boldsymbol{\eta}}{\partial \xi_{2}}\right)}{\left\|\mathbf{g}_{10}\right\|\left\|\mathbf{g}_{20}\right\|} \\
& \left.-\cos \left(\theta_{0}\right) \frac{\mathbf{g}_{10}}{\left\|\mathbf{g}_{10}\right\|^{2}} \cdot \frac{\partial \boldsymbol{\eta}}{\partial \xi_{1}}+\frac{\mathbf{g}_{20}}{\left\|\mathbf{g}_{20}\right\|^{2}} \cdot \frac{\partial \boldsymbol{\eta}}{\partial \xi_{2}}\right)
\end{aligned}
$$


We note $\theta=\left(\mathbf{g}_{1}, \mathbf{g}_{2}\right)=\theta_{0}+\gamma(\boldsymbol{\eta})$ the angle between warp and weft yarns in the virtual configuration. We have

$\cos \left(\theta_{0}\right)-\cos (\theta) \simeq \sin \left(\theta_{0}\right) \gamma(\boldsymbol{\eta})$

from where

$$
\begin{aligned}
\gamma(\boldsymbol{\eta}) \sin \left(\theta_{0}\right) \simeq & -\frac{\left(\mathbf{g}_{20} \cdot \frac{\partial \boldsymbol{\eta}}{\partial \xi_{1}}+\mathbf{g}_{10} \cdot \frac{\partial \boldsymbol{\eta}}{\partial \xi_{2}}\right)}{\left\|\mathbf{g}_{10}\right\|\left\|\mathbf{g}_{20}\right\|} \\
& \left.+\cos \left(\theta_{0}\right) \frac{\mathbf{g}_{10}}{\left\|\mathbf{g}_{10}\right\|^{2}} \cdot \frac{\partial \boldsymbol{\eta}}{\partial \xi_{1}}+\frac{\mathbf{g}_{20}}{\left\|\mathbf{g}_{20}\right\|^{2}} \cdot \frac{\partial \boldsymbol{\eta}}{\partial \xi_{2}}\right)
\end{aligned}
$$

$\gamma(\boldsymbol{\eta})$ is obtained as

$$
\begin{aligned}
\gamma(\boldsymbol{\eta})= & \frac{\partial \boldsymbol{\eta}}{\partial \xi_{1}} \cdot\left[\cot g \theta_{0} \frac{\mathbf{g}_{10}}{\left\|\mathbf{g}_{10}\right\|^{2}}-\frac{\mathbf{g}_{20}}{\sin \theta_{0}\left\|\mathbf{g}_{10}\right\|\left\|\mathbf{g}_{20}\right\|}\right] \\
& +\frac{\partial \boldsymbol{\eta}}{\partial \xi_{2}} \cdot\left[\cot g \theta_{0} \frac{\mathbf{g}_{20}}{\left\|\mathbf{g}_{20}\right\|^{2}}-\frac{\mathbf{g}_{10}}{\sin \theta_{0}\left\|\mathbf{g}_{10}\right\|\left\|\mathbf{g}_{20}\right\|}\right]
\end{aligned}
$$

$\gamma(\boldsymbol{\eta})$ can be written as the product of the virtual node displacements vector and a column matrix $\mathbf{B}_{\gamma}$

$\gamma(\boldsymbol{\eta})=\mathbf{B}_{\gamma} \boldsymbol{\eta}=B_{\gamma s} \eta_{s}$

With

$$
\begin{aligned}
B_{\gamma s}= & \frac{\partial N^{\mathrm{k}}}{\partial \xi_{1}}\left[\cot g \theta \frac{\left(\mathbf{g}_{1}\right)_{m}}{\left\|\mathbf{g}_{1}\right\|^{2}}-\frac{\left(\mathbf{g}_{2}\right)_{m}}{\sin \theta\left\|\mathbf{g}_{1}\right\|\left\|\mathbf{g}_{2}\right\|}\right] \\
& +\frac{\partial N^{\mathrm{k}}}{\partial \xi_{2}}\left[\cot g \theta \frac{\left(\mathbf{g}_{2}\right)_{m}}{\left\|\mathbf{g}_{2}\right\|^{2}}-\frac{\left(\mathbf{g}_{1}\right)_{m}}{\sin \theta\left\|\mathbf{g}_{1}\right\|\left\|\mathbf{g}_{2}\right\|}\right]
\end{aligned}
$$

where $k=\operatorname{Int}\left(\frac{s+2}{3}\right), m=s-3(k-1)$ and $s$ the range of the degree of freedom in the element.

The new expression of the virtual work of the interior loads after finite element approximation is

$$
\begin{aligned}
T_{\text {int }}^{\mathrm{e}}(\boldsymbol{\eta})= & \eta_{s} \sum_{p=1}^{n_{t}} \frac{1}{\left\|\mathbf{g}_{1}\right\|}{ }^{p} B_{11 s}^{p} T^{11}+\sum_{p=1}^{n_{c}} \frac{1}{\left\|\mathbf{g}_{2}\right\|}{ }^{p} B_{22 s}^{p} T^{22} \\
& \left.+\sum_{p=1}^{n_{t} n_{c}}{ }^{p} C^{p} B_{\gamma s}\right)=\eta_{s}\left(\mathbf{F}_{\text {int }}^{\mathrm{e}}\right)_{s}
\end{aligned}
$$

It is possible to replace the summation on all the warp and weft crossovers by a summation on only 4 crossovers. The positions of these particular points depend on the numbers of yarns in warp and weft directions of the element. The interior loads vector is computed using the following expression:

$$
\begin{aligned}
\left(F_{\mathrm{int}}\right)_{s}= & \sum_{p=1}^{2} n_{c}{ }^{p} B_{11 s} T^{11} \frac{1}{\left\|\mathbf{g}_{1}\right\|}+\sum_{p=1}^{2} n_{t} B_{22 s} T^{22} \frac{1}{\left\|\mathbf{g}_{2}\right\|} \\
& +\frac{1}{4} \sum_{p=1}^{4} n_{c} n_{t} B_{\gamma s}{ }^{p} C
\end{aligned}
$$

In this equation $B_{\gamma s}$ and ${ }^{p} C$ are calculated at the four positions $p$ given by Eq. (13).
Eq. (33) provides an approximate computation of the interior loads due to shearing (contrary to the tension which is calculated in an exact manner).

\section{Validation and forming simulations}

\subsection{Verification of the discrete quadrature for shear}

The error made in the computation of the shear contribution is evaluated in function of the fibres density in the finite element. An hourglass shape is considered (Fig. 10). The error is maximal for this particular element geometry. This shape is uncommon in practice because the yarns of a fabric do not usually stretch out much and consequently the deformed element is close to a lozenge. In a lozenge the warp and weft yarn angles are equal, so the discrete quadrature provides an exact computation.

An exact computation of the matrix $\mathbf{B}_{\gamma}$ is obtained by summation on all the crossovers. It is compared with the results obtained using the discrete quadrature. Fig. 11 provides the evolution of the maximum of the relative error committed on the shear contribution in the terms of the interior loads vector according to the shear angle $\gamma$ for different warp and weft element densities. The relative error is very weak for angles lower then $0.5 \mathrm{rd}$ and remains small for large angles $(0.9 \mathrm{rd})$. The error is equal to zero for two yarns and remains small when the number of yarn in the element increases. We can conclude that the approximation made to compute the sum on all warp and weft intersections provided a good result with a very weak error. It permits an important gain in computational time.

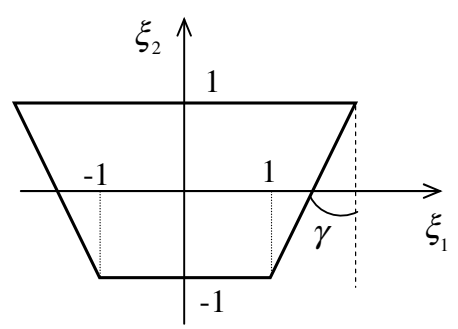

Fig. 10. Hour glass shape element.

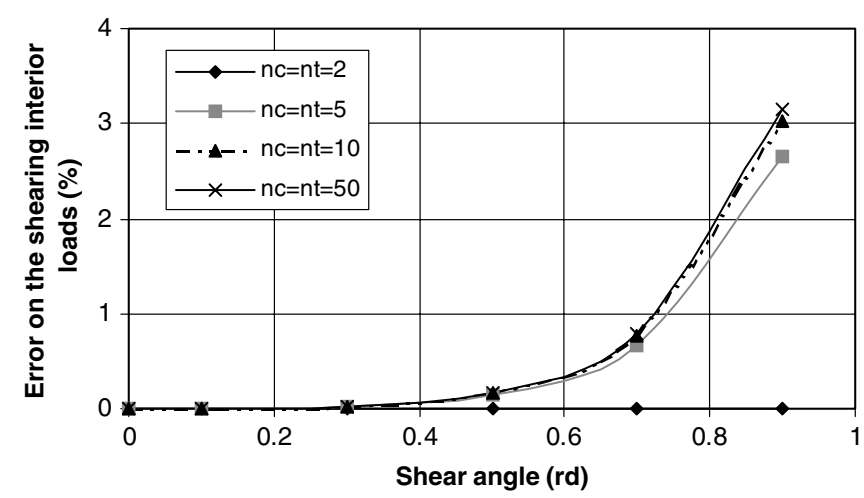

Fig. 11. Error on the shearing interior loads. 


\subsection{Element validation}

It is first necessary to verify the approximation of $\gamma(\boldsymbol{\eta})$ given by the expression (29) for time steps used in practical explicit computations. In this goal, several deformation cases are considered. For these cases, the approached values of the angle $\theta$ between warp and weft directions are compared with the exact values.

\subsubsection{Traction}

A unit side square element is submitted to a $100 \%$ elongation in direction 1 (Fig. 12(a)). The computation needs 3400 steps. The results show that the angle $\theta$ between warp and weft remains constant and equal to $90^{\circ}$.

\subsubsection{Shearing}

The same element is now submitted to simple shear (Fig. 12(b)). The computation needs 3400 steps. Fig. 13, the two curves show the variation of the angle between warp and weft yarns calculated by the approximated value given by Eq. (29) and by an exact computation. The two curves are perfectly superimposed. Fig. 14 shows the difference between the two previous curves. The error remains very small. The computation of the angle between warp and weft from the expression (29) is therefore satisfactory.

\subsection{Pure shear test}

An analytical solution for the pure shear test applied on a square elementary cell of unit side containing one warp

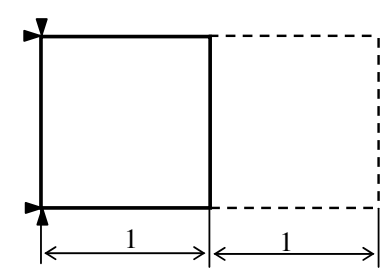

a

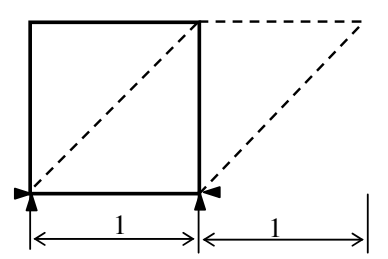

b
Fig. 12. Elementary tests: (a) traction; (b) shear.

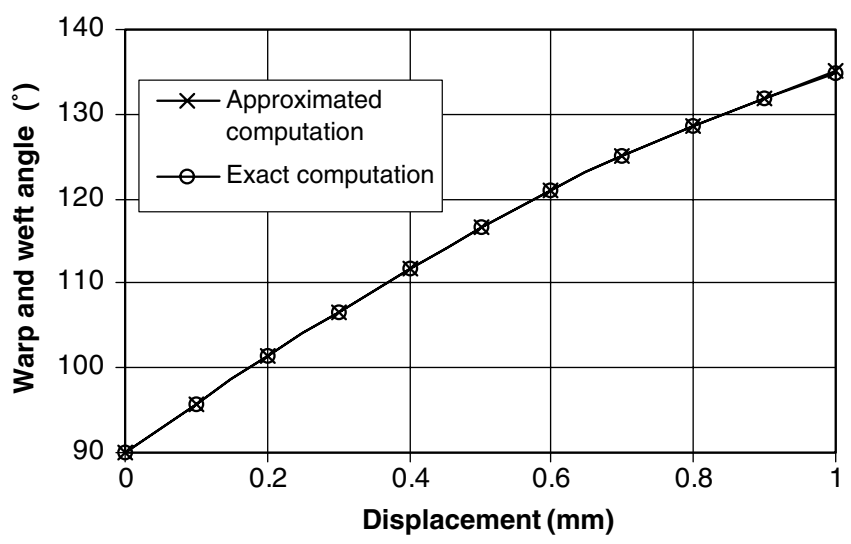

Fig. 13. Angle variation between warp and weft yarns in the case of a shear test.

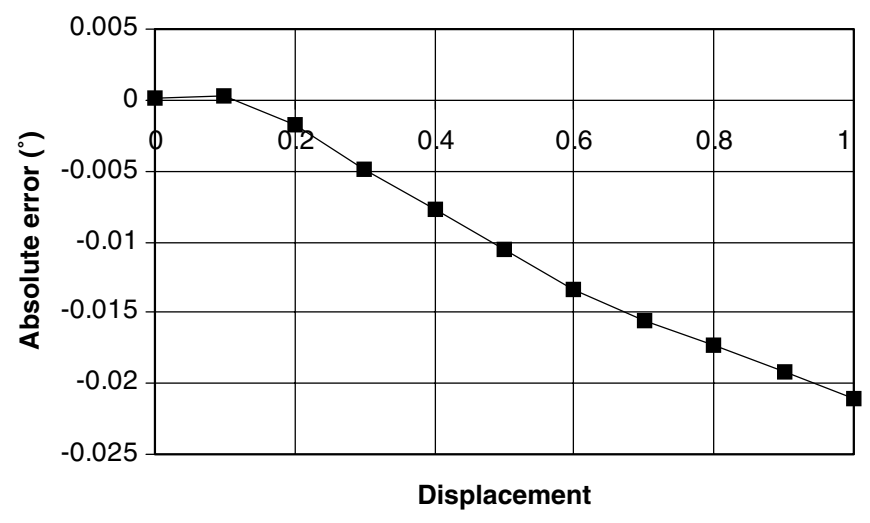

Fig. 14. Absolute error committed on the computation of the angle between warp and weft yarns.

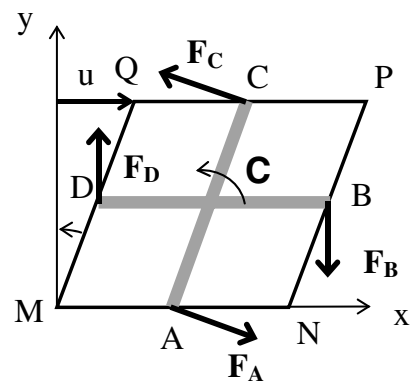

Fig. 15. Pure shear test on a unit woven cell.

and one weft yarns has been developed (Fig. 15). The nodal force components issued from the analytical solution and from the proposed numerical approach, are compared according to the shear angle. We suppose that a torque $C$ proportional to the shear angle is applied at the warp and weft intersection.

$C(\gamma)=k \gamma$

where $k$ is the shear rigidity.

When bringing back the loads $\mathbf{F}_{\mathrm{A}}, \mathbf{F}_{\mathrm{B}}, \mathbf{F}_{\mathrm{C}}$ and $\mathbf{F}_{\mathrm{D}}$ to the nodes $\mathrm{M}, \mathrm{N}, \mathrm{P}$ and $\mathrm{Q}$, we get

$$
\begin{cases}F_{1}^{x}=-k \gamma \cos \gamma, & F_{1}^{y}=-k \gamma(1-\sin \gamma) \\ F_{2}^{x}=-k \gamma \cos \gamma, & F_{2}^{y}=k \gamma(1+\sin \gamma) \\ F_{3}^{x}=k \gamma \cos \gamma, & F_{3}^{y}=k \gamma(\sin \gamma-1) \\ F_{4}^{x}=k \gamma \cos \gamma, & F_{4}^{y}=-k \gamma(1+\sin \gamma)\end{cases}
$$

with $\gamma=\arcsin (u)$, where $u$ is the horizontal displacement of the points $\mathrm{C}, \mathrm{P}$ and $\mathrm{Q}$.

Figs. 16-18 show the theoretical nodal loads and those obtained from the developed numerical model for different values of the fibre tensile rigidities. The rigidity of the yarn is denoted $E(\mathrm{~N} / \mathrm{mm})$. When it increases the computed solution differs slightly from the analytic solution. This can be explained by the approximations on the node speeds prescribed in $y$-direction. 


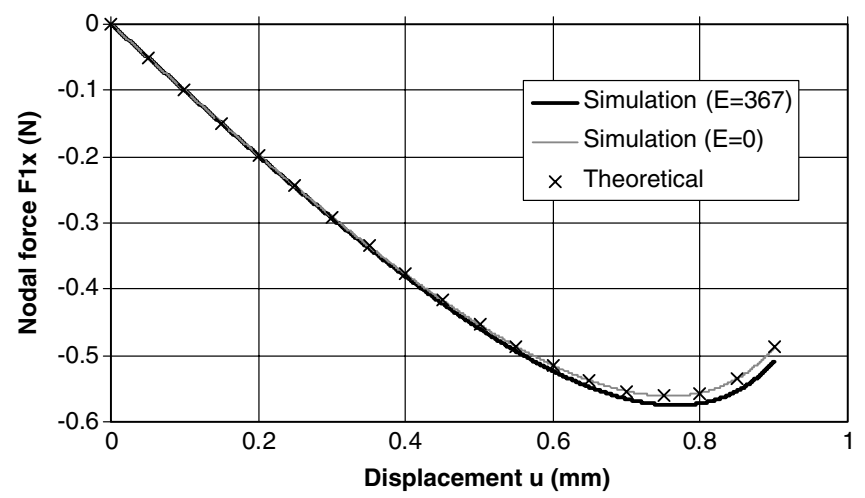

Fig. 16. Load component at point $\mathrm{M}$ in the $x$-direction versus displacement.

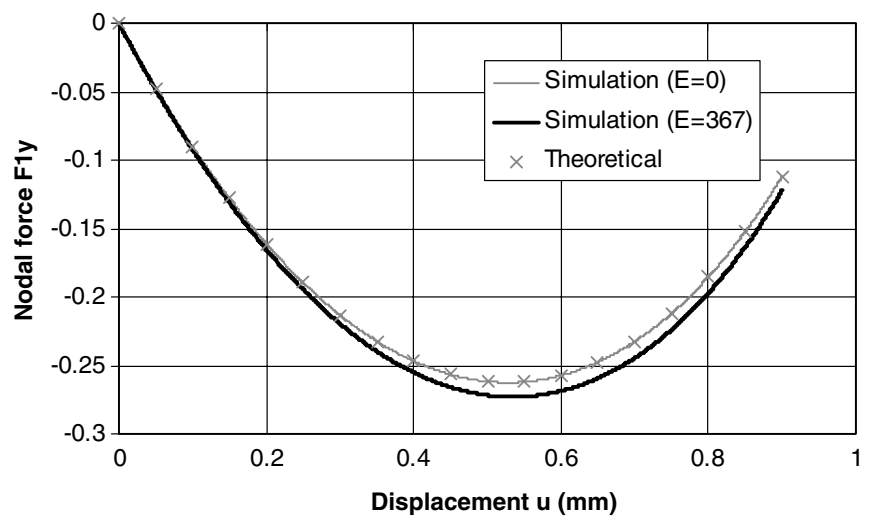

Fig. 17. Load component at point $\mathrm{M}$ in the $y$-direction versus displacement.

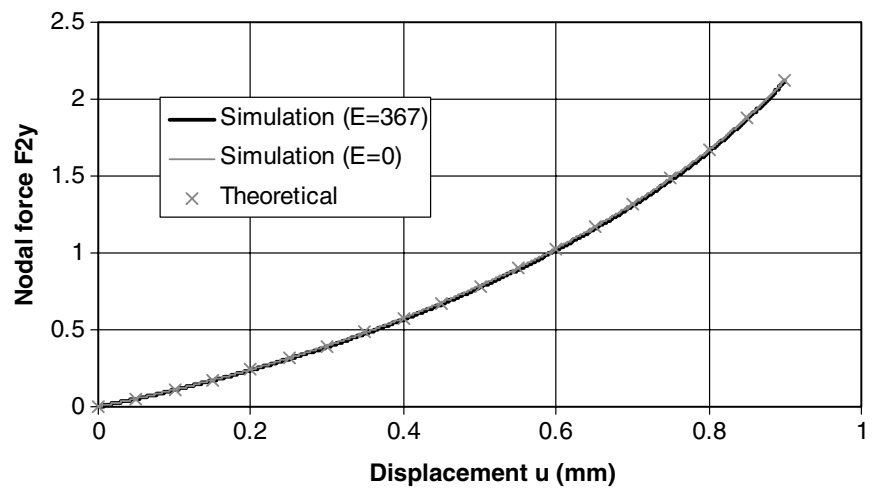

Fig. 18. Load component at point $\mathrm{N}$ in the $x$-direction versus displacement.

\subsection{Simulation of the picture frame test}

A picture frame test is equivalent to a pure shear test plus a rigid body rotation. This test has been simulated by using the presented approach and for different meshes (Fig. 19).

Fig. 20 shows the variation of the force calculated by our model according to the displacement (for different meshes: 1 element, 4 elements and 16 elements) and the
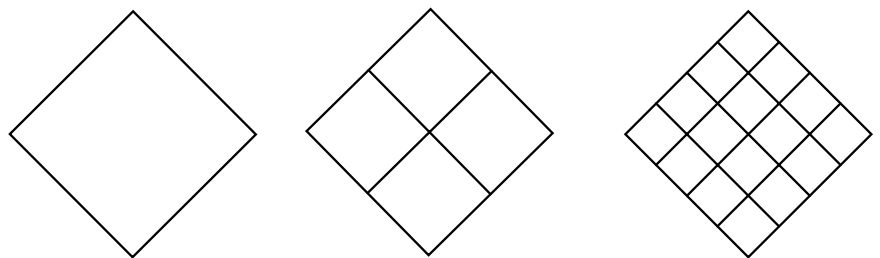

Fig. 19. Meshes used for the picture frame test simulation.

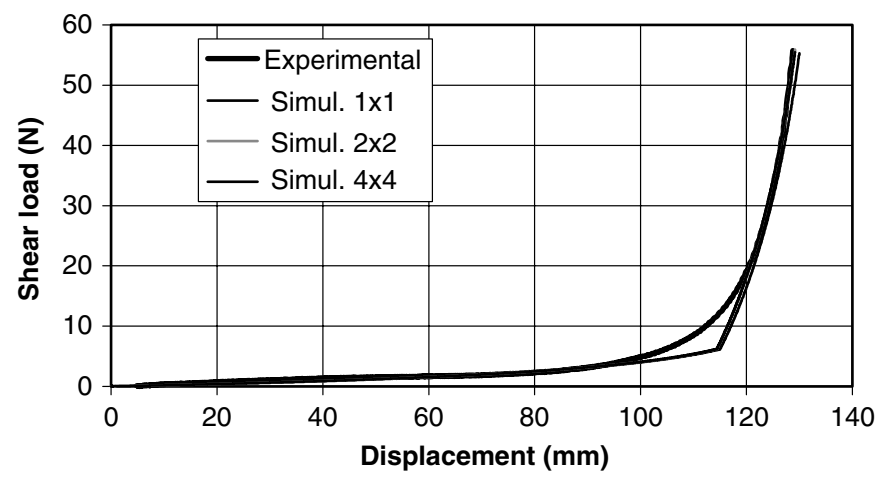

Fig. 20. Computed load and experimental load according to the displacement.

experimental force measured on the traction machine. One can notice that the calculated force is insensitive to the mesh density.

The velocity imposed on the boundary nodes of the frame evolves in a progressive manner (class $\mathrm{C} 1$ in relation to the time). In the contrary case, the initial knock induces strong oscillations (see Fig. 21).

\subsection{Square box deep drawing}

The test presented Fig. 22 is a classic benchmark for sheet forming. It has been proposed at the Numisheet 93 conference [33]. For the woven sheet forming, this test is severe because it leads to large variations of angle between warp and weft in the radius of the square box. If these radius become small, we get some warp and weft angle

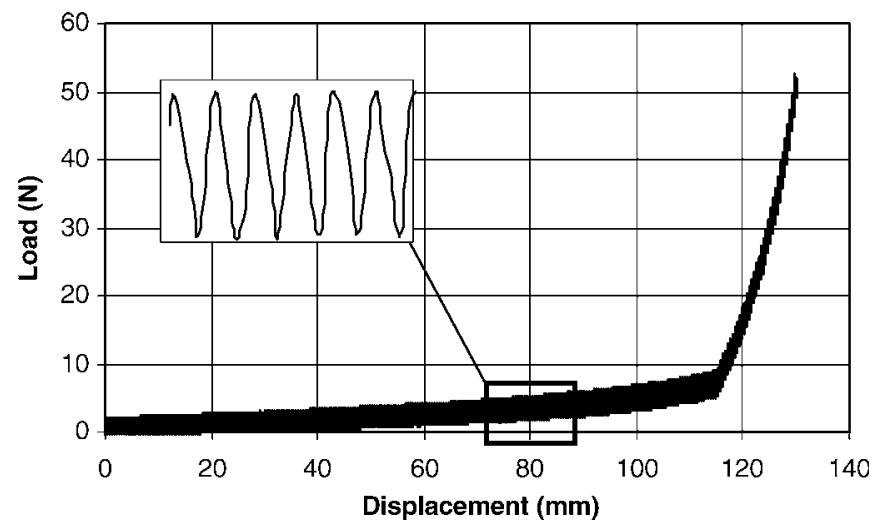

Fig. 21. Computed load when the prescribed velocity is constant. 


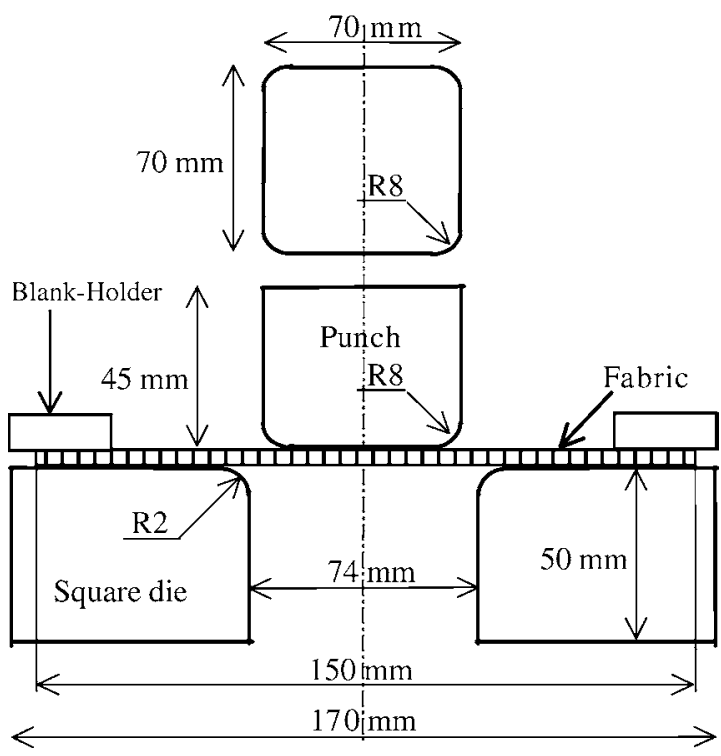

Fig. 22. Square box deep drawing: geometry of the tools.

distortions superior to the limit angle of the fabric. The sheet forming simulation of a glass plain weave (T6 tested in Section 2) is achieved using the tension only approach (Eq. (4)) and the tension-shear approach (Eq. (17)). Fig. 23 presents the two deformed shapes of the reinforcement at the end of the forming. Due to the strongly double curved character of the formed surface, the shear limit
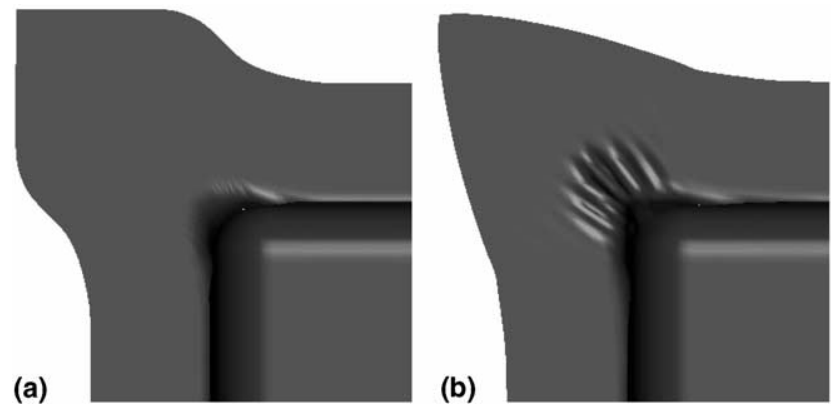

Fig. 23. Final shape of the fabric: approach in tension (a) and approach in tension + shear $(b)$. angle of the fabric is exceeded. There is no wrinkling in the tension only solution because there is no source of instability in this case. On the other hand some wrinkles are developed in the second approach. They are due to the fabric shear locking that leads to out of plan solutions in order to reduce the shear strain energy. Fig. 24 shows that the shear angles are reduced when shearing is taken into account (maximum shear angle is $48^{\circ}$ ). Nevertheless, the tension approach also detects that the shear angle exceeds the locking angle in some places. Wrinkles appearance is detected but those wrinkles are not described. The contribution of the shear in the approach is mainly the description of the deformed shape after wrinkling.

\subsection{Hemispherical forming of an unbalanced fabric}

The hemispherical forming of a $2 \times 2$ nylon twill is analysed in this section. This fabric is used in automotive industry [31]. It presents a very unbalanced tensile behaviour in warp and weft direction (Fig. 25). The warp rigidity is $50 \mathrm{~N} / \mathrm{yarn}$ and the weft rigidity is $0.2 \mathrm{~N} / \mathrm{yarn}$. The shear behaviour of this fabric has been experimentally analysed by the picture frame test. Its rigidity has been approached by two straight segments, whose slopes are $k_{1}=0.03 \mathrm{mmN} / \mathrm{rd}$ and $k_{2}=0.095 \mathrm{mmN} / \mathrm{rd}$ with a critical shear angle $\gamma_{c}=0.5 \mathrm{rd}$.

Tests of hemispheric sheet forming have been achieved by Dumont in the S3MEM composites laboratory of the University of Nottingham [31,34]. It is a classic case of a double curved geometry [24,35-37] that presents the advantage to have regular change of curvatures while producing strong distortions for a sufficiently deep forming [38]. This forming process has been simulated using the two approaches (tension only, tension + shear). The results of these two simulations as well as the experimental final shape are shown Fig. 26. The experimental deformed shape is very different in warp and weft direction. The warp direction (vertical in Fig. 26) corresponding to the most rigid yarns, shows an important sliding of fabric in the matrix. In the other (weft) direction, there is no visible sliding. The yarns are strongly stretched. At the summit of the hemisphere, an initially square quadrilateral becomes a

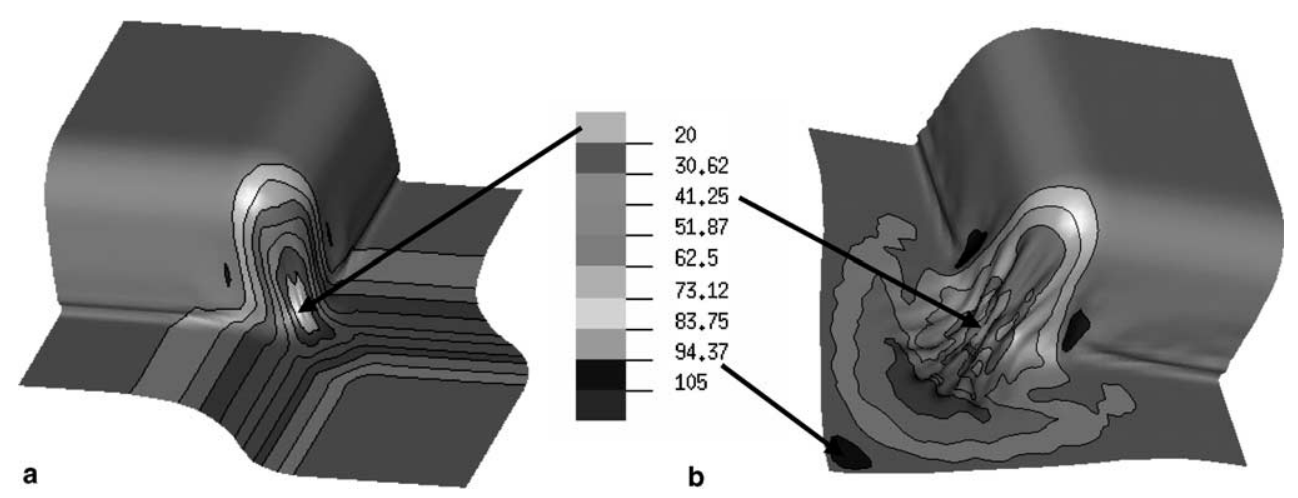

Fig. 24. Angle between warp and weft yarns: approach in tension (a) and approach in tension + shear (b). 


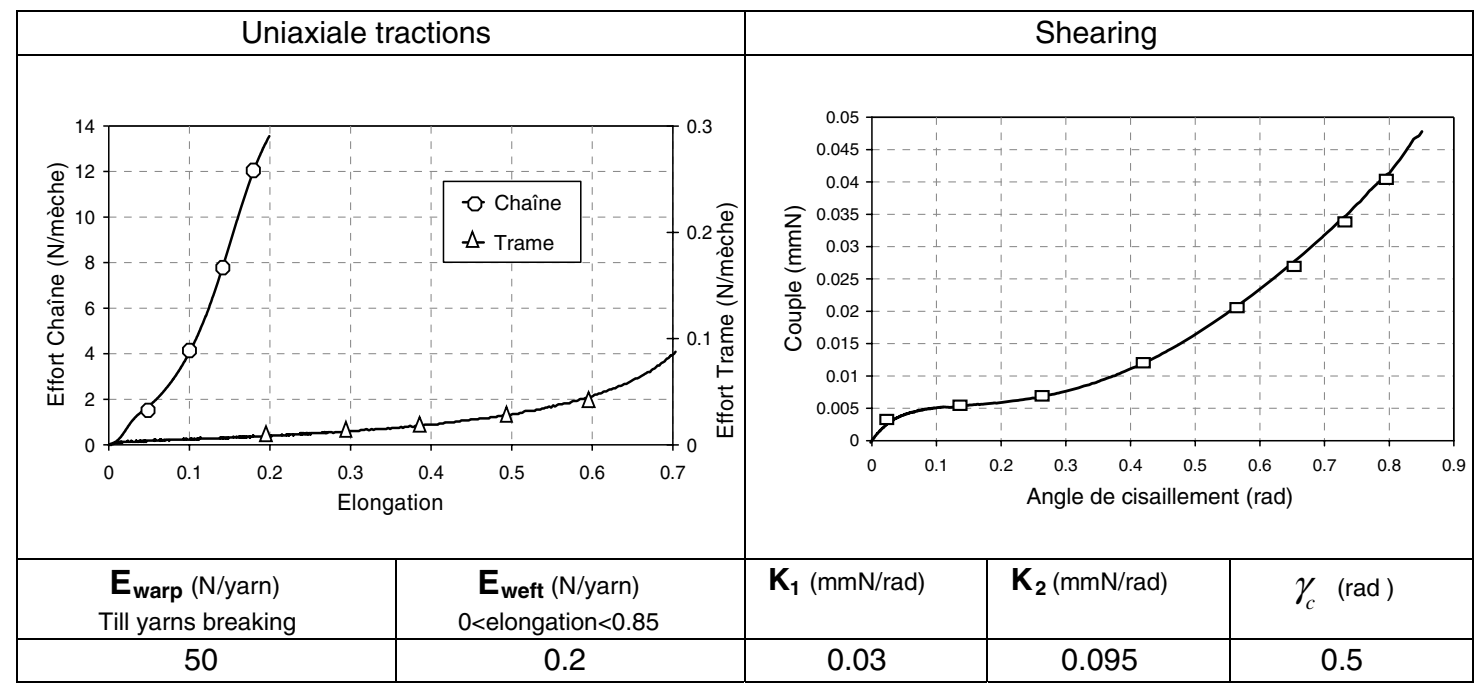

Fig. 25. Characteristics of the woven reinforcement, in tension (warp and weft direction), and in shear.
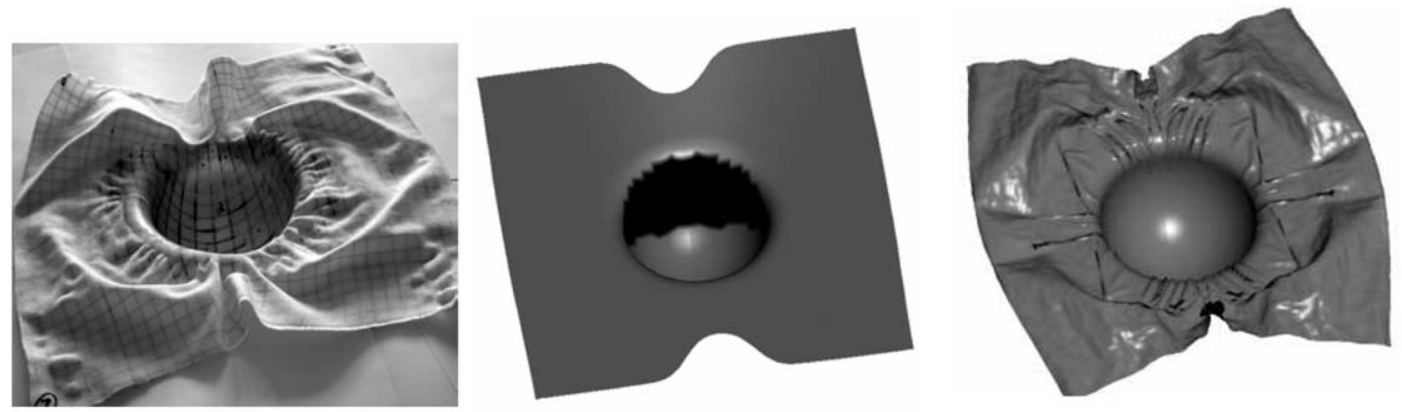

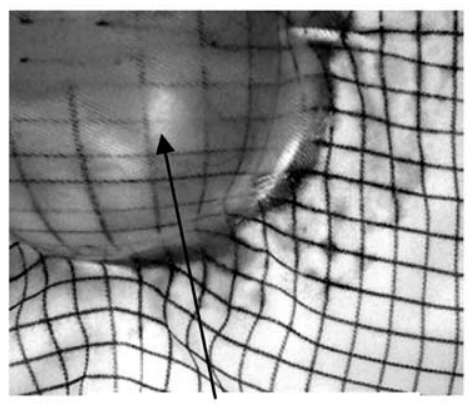

$L$ Weft/L Warp $=1.80$

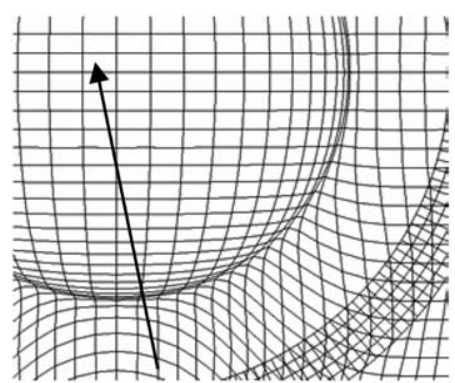

$\mathrm{L}$ Weft/L Warp $=1.87$

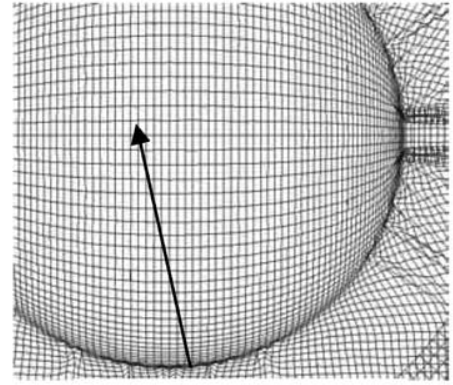

$\mathrm{L}$ Weft/L Warp $=1.83$

b

Fig. 26. Deformed shape after the hemispheric forming in the experimental case (a), simulation in tension only (b) and in tension + shear (c).

rectangle with a side ratio equal to 1.8 (Fig. 26(a)). Both simulations give value of this ratio close to this value (Fig. 26(b) and (c)). The deformation of the hemispherical part is well computed by both approaches.

The asymmetry of the deformed shape in warp and weft direction is obtained by both approaches. The main difference concerns the wrinkles. In tension + shear approach, the shear strain energy leads to the appearance of wrinkles in the plane region of the preform (contrary to the approach in tension only). Their shapes are in good agreement with those of the experimental preform (Fig. 26(a) and (c)). There is no wrinkle in the hemispherical zone and the two approaches give close results in this region. But there are regions where the shear angle is higher than the shear limit angle. When the shear energy is taken into account, the minimisation of the total deformation energy leads to an out of plane solution i.e. to the wrinkles (Fig. 26(c)). The contribution of the shear behaviour is mainly in the description of the state after wrinkle appearance. In this stage, the relative rotations between warp and weft yarns are reduced. For instance the maximum shear angle is $37^{\circ}$ in Fig. 26(c) instead of $50^{\circ}$ Fig. 26(b). This 
value ( $37^{\circ}$ in case of (tension + shear) analysis) is in good agreement with angles measured on experimental parts $\left(37^{\circ}\right)$. It can be observed that the mesh used Fig. 26(c) (tension + shear) is finer than those used in 26(b) (tension only). These 1 meshes are used because they are sufficient, i.e. that their refinement does not change significantly the solution. The needed size is much finer in the case tension + shear because the wrinkles that appear in this case need elements of a time much smaller than those of these wrinkles in order to describe then correctly. The explicit scheme permits to obtain them without any perturbation method or initial imperfection.

\section{Conclusion}

The objective of this work is to show the influence of the fabric shear rigidity on the prediction of the final shape of the woven reinforcement after forming. It has been observed according to the experimental study of shearing with a picture frame, that the principal mode of shear deformation of the woven reinforcement is the relative rotation between the warp yarns and the weft ones. Consequently, we supposed the existence of a resistive torque applied to the level of the warp and weft intersections varying according to the shear angle. The evolution of the shear rigidity according to the shear angle has been deduced. This shear behaviour has been introduced in the woven finite element without addition of new degrees of freedom. This approach gives better results than those only based on tension properties when the shear limit angle is exceeded and when consequently some wrinkles appear. In these cases, deformations modes are obtained in order to reduce the shear angle (that corresponds to a strong stiffness in this situation). This has been shown in a square box and in a hemispherical forming cases. A next goal will be to investigate the importance of the yarn bending rigidity when the woven reinforcement is thick.

\section{Acknowledgements}

The authors acknowledge the support provided by the EADS aeronautical company and PSA automotive company.

\section{References}

[1] Gauvin R, Trochu F, Lemenn Y, Diallo L. Permeability measurement and flow simulation through fibre reinforcement. Polym Compos 1996;17:34 42.

[2] Bergsma OK, Huisman J. Deep drawing of fabric reinforced thermoplastics. In: Brebbia CA et al., editors. Proceedings of the 2nd int conf on computer aided design in composite material technology. Springer-Verlag; 1988. p. 323-33.

[3] Van Der Ween F. Algorithms for draping fabrics on doubly curved surfaces. Int J Numer Method Eng 1991;31:1414-26.

[4] Borouchaki H, Cherouat A. Une nouvelle approche pour le drappage des structures composites. Rev Comp Mat Avanc 2002;32:407-22.

[5] Rogers TG. Rheological characterization of anisotropic materials. Composites 1989;20(1).
[6] Xue P, Peng XQ, Cao J. A non-orthogonal constitutive model for characterizing woven composite. Composites Part A 2003;34:183-93.

[7] Hagège $B$, Boisse P, Billoët JL. Analysis and simulation of the constitutive behavior of fibrous reinforcements. In: Proceedings of Esaform7 conf, Trondheim, avril 2004, p. 317-20.

[8] Kawabata S, Niwa M, Kawai H. The finite-deformation theory of plain-weave fabrics. Part I: The biaxial deformation theory. J Textile Inst 1973;64-2:21-46.

[9] Kawabata S. Nonlinear mechanics of woven and knitted materials. Chou TW, Ko FK, editors. Textile structural composites, vol. 3. Elsevier; 1989. p. 67-116.

[10] Buet-Gautier K, Analyse et simulation du comportement mécanique des renforts composites tissés, Thèse de doctorat, Mécanique des solides, Université d'Orléans, France, 1998, 125p.

[11] Buet-Gautier K, Boisse P. Experimental analysis and modeling of biaxial mechanical behavior of woven composite reinforcements. Exp Mech 2001;41(3):260-9.

[12] Kawabata S, Niwa M, Kawai H. The finite-deformation theory of plain-weave fabrics. Part III: The shear-deformation theory. J Textile Inst 1973;63:62-85.

[13] Yick KL, Cheng KPS, Dhingra RC, How YL. Comparison of mechanical properties of shirting materials measured on the KES-F and FAST instruments. Textile Res J 1996;66(10):622-33.

[14] Wang J, Page JR, Paton R. Experimental investigation of the draping properties of reinforcement fabrics. Compos Sci Technol 1998;58(2): 229-37.

[15] Hofstee J, De Boer H, Van Keulen F. Elastic stiffness analysis of a thermo-formed plain weave fabric composite. Part I: Geometry. Compos Sci Technol 2000;60(7):1041-53.

[16] Sidhu RMJS, Averill RC, Riaz M, Pourboghrat F. Finite element analysis of textile composite preform stamping. Compos Struct 2001; 52(3-4):483-97.

[17] McBride TM, Chen J. Unit-cell geometry in plain-weave fabrics during shear deformations. Compos Sci Technol 1997;57(3):345-51.

[18] McGuinness GB, Bradaigh CMO. Development of rheological models for forming flows and picture-frame shear testing of fabric reinforced thermoplastic sheets. J Non-Newtonian Fluid Mech 1997; 73(1-2):1-28.

[19] McGuinness GB, Bradaigh CMO. Characterization of thermoplastic composite melts in rhombus-shear: the picture-frame experiment. Composites Part A 1998;29(1-2):115-32.

[20] Long AC. Characterisation and modelling of fabric deformation during forming of textile composites. Int J Forming Process 2002;4: 285-301.

[21] Hivet G, Dumont F, Launay J. Maurel V, Vacher P, Boisse P, Optical analysis of woven fabric's shear behaviour. In: Proceedings of Esaform7 conf, Trondheim, avril 2004, p. 353-6.

[22] Sutton M, Wolters WJ, Peters WH, Ranson WF, McNeill SR. Determination of displacements using an improved digital correlation method. Image Vis Comput 1983;1(3):133-9.

[23] Vacher P, Dumoulin S, Arrieux R. Determination of the forming limit diagram from local measurement using digital image analysis. Int J Forming Process 1999;2(3-4):395-408.

[24] Boisse P, Cherouat A, Gelin JC, Sabhi H. Experimental study and finite element simulation of a glass fibre fabric shaping process. Polym Compos 1995; 16(1):83-95.

[25] Boisse P, Borr M, Buet K, Cherouat A. Finite element simulation of textile composite forming including the biaxial fabric behaviour. Composites Part B 1997;28:453-64.

[26] Boisse P. Modèles mécaniques et numériques pour l'analyse nonlinéaire des structures minces, Mémoire d'habilitation à diriger des recherches. Besançon, France, 1994.

[27] Gelin JC, Cherouat H, Boisse P, Sabhi H. Manufacture of thin composite structures by the RTM process : numerical simulation of the shaping operation. Compos Sci Technol 1996;56:711-8.

[28] Boisse P, Gasser A, Hivet G. Analyses of fabric tensile behaviour: determination of the biaxial tension-strain surfaces and their use in forming simulations. Composites Part A 2001;32:1395-414. 
[29] Boisse P, Daniel JL, Hivet G, Soulat D. A simplified explicit approach for simulations of fibre fabric deformation during manufacturing preforms for R.T.M. process. Int J Forming Process 2001; 3(3-4):351-3.

[30] Yu X, Ye L, Mai Y-W. Finite element spurious wrinkles on the thermoforming simulation of woven fabric reinforced composites. In: Proceedings of the int conf ESAFORM7, Trondheim, April 2004, p. 325-8.

[31] Dumont F. Expérimentations et modèles de comportement de renforts de composites tissés, Thèse de doctorat, Université de Paris VI, France, 2003.

[32] Zouari B, Dumont F, Daniel JL, Boisse P. Studies of woven fabric shearing by optical method and implementation in a finite element program. In: Proceedings of Esaform6, 2003, p. 875-8.

[33] Makinouchi A, Nakamachi E, Onate E, Wagoner RH, editors. Numisheet 93, Numerical simulation of 3-D sheet metal forming processes - verification of simulation with experiments, Japan, 1993.
[34] Daniel JL, Soulat D, Dumont F, Zouari B, Boisse P, Long AC. Forming of a very unbalanced fabric. Experiment and simulation. Int J Forming Process 2003;6(3-4):465-80.

[35] Hu J, Jiang Y. Modeling formability of multiaxial warp knitted fabrics on a hemisphere. Composites Part A 2002;33(5):725-34.

[36] Potter K. Beyond the pin-jointed net: maximising the deformability of aligned continuous fibre reinforcements. Composites Part A 2002; 33(5):677-86.

[37] Boisse P, Soulat D, Daniel JL. Numerical analysis of continuous fiber composite forming. In: Topping BHV, Mota CA, editors. Progress in computational structure technology, Invited lectures of CST 2004, Soares, Saxe-Coburg publications; 2004. p. 311-26.

[38] Rudd CD, Turner MR, Long AC, Middleton V. Tow placement studies for liquid composite moulding. Composites Part A 1999;30(9): 1105-21. 